\title{
Transcatheter arterial embolization as an effective treatment for spontaneous inferior epigastric artery bleeding caused by anticoagulant therapy: Case reports
}

\author{
YEN-CHENG CHEN $^{1}$, CHIEN-LIN HUANG ${ }^{2}$, MING-CHEN SHIH ${ }^{3}$ and JAW-YUAN WANG ${ }^{1,4-6}$ \\ ${ }^{1}$ Division of Colorectal Surgery, and ${ }^{2}$ Division of Cardiovascular Surgery, Department of Surgery, \\ Kaohsiung Medical University Chung-Ho Memorial Hospital, Kaohsiung Medical University; ${ }^{3}$ Department of Radiology, \\ Kaohsiung Medical University Chung-Ho Memorial Hospital, Kaohsiung Medical University; ${ }^{4}$ Department of Surgery, \\ Faculty of Medicine, ${ }^{5}$ Graduate Institute of Clinical Medicine, and ${ }^{6}$ Graduate Institute of Medicine, \\ College of Medicine, Kaohsiung Medical University, Kaohsiung 807, Taiwan, R.O.C.
}

Received June 7, 2020; Accepted September 17, 2020

DOI: 10.3892/wasj.2020.69

\begin{abstract}
Spontaneous inferior epigastric artery hemorrhage (SIEAH) is a rare, yet potentially fatal condition. In some cases, the failure of hemostasis can result in hypovolemic shock or even death. Anticoagulant therapy is strongly associated with patient mortality. The present study describes two cases of SIEAH that were successfully dealt with using transcatheter arterial embolization (TAE). The standard use of anticoagulant or antiplatelet agents may induce episodes of severe bleeding, such as SIEAH. Although the condition is rare, it can potentially be life-threatening. The diagnosis mainly depends on computed tomography (CT) findings. With hemodynamic instability, surgical intervention is the most standard treatment. However, in patients with severe comorbidities, old age, or other associated injuries, several post-operative complications may occur. To date, data supporting that TAE is more suitable than the traditional open surgery are lacking. In some selected patients, compared with open surgery, TAE can be a relatively safe and effective modality for the termination of bleeding.
\end{abstract}

Correspondence to: Professor Jaw-Yuan Wang, Division of Colorectal Surgery, Department of Surgery, Kaohsiung Medical University Chung-Ho Memorial Hospital, Kaohsiung Medical University, 100 Tzyou 1st Road, Kaohsiung 807, Taiwan, R.O.C.

E-mail: cy614112@ms14.hinet.net; jawyuanwang@gmail.com

Abbreviations: SIEAH, spontaneous inferior epigastric artery hemorrhage; RSH, rectus sheath hematoma; TAE, transcatheter arterial embolization; IEA, inferior epigastric artery; PT, prothrombin time; INR, international normalized ratio; CT, computed tomography

Key words: spontaneous hemorrhage, inferior epigastric artery, anticoagulant therapy, transcatheter arterial embolization, superselective catheterization

\section{Introduction}

Spontaneous inferior epigastric artery hemorrhage (SIEAH) is a rare condition and can lead to rectus sheath hematoma (RSH), and may thus prove potentially fatal condition (1-5). Bleeding can lead to hematoma and tamponade; therefore, this episode may be self-limited (2,6-8). However, in some cases, hemorrhaging can result in hypovolemic shock or even death. Anticoagulant therapy has been reported to be a risk factor of mortality $(1,3,4)$. Other risk factors of SIEAH include hypertension, pregnancy, atherosclerosis, obesity, the use of corticosteroids, thrombophilia and leukemia $(1,5,8)$. Initially, conservative treatment, including blood transfusion, anticoagulation therapy and coagulation parameter correction, is the most commonly used treatment $(1,2,4,9,10)$. However, in some patients with an unstable hemodynamic status, invasive intervention is indicated $(2,4,9,10)$. In some selected cases, compared with open surgery, transcatheter arterial embolization (TAE) can be a relatively safe and effective modality for the termination of bleeding $(2,9,10)$. The present study describes two cases that underwent anticoagulant therapy and developed spontaneous inferior epigastric artery (IEA) bleeding. Both cases were successfully dealt with using TAE.

\section{Case reports}

Case 1. A 72-year-old male subject with hypertension and coronary artery disease was diagnosed with sigmoid colon cancer (clinical stage, cT3N1M0; stage IIIB), and sigmoid colectomy was subsequently performed. A few hours post-surgery, the patient was diagnosed with acute myocardial infarction. Considering the fasting status following colorectal surgery, a heparin pump was initially applied and this was then switched to oral antiplatelet agents (clopidogrel, $75 \mathrm{mg}$ daily; aspirin, $100 \mathrm{mg}$ daily).

On post-operative day 10 , the patient suffered from bleeding from the operative wound with hypovolemic shock. The hemoglobin level decreased from 12.0 to $9.2 \mathrm{~g} / \mathrm{dl}$. Laboratory data revealed a normal prothrombin time (PT) and the international normalized ratio (INR) was 1.03 (range, 0.85-1.15). 
Table I. Summary of the two cases of SIEAH.

\begin{tabular}{llllll}
\hline Age $($ years)/sex & Underlying disease & Anticoagulant & Laboratory data & Bleeding point & Embolization \\
\hline $72 /$ male & Acute myocardial & $\begin{array}{l}\text { Heparin, clopidogrel, } \\
\text { infarction }\end{array}$ & Normal & Left IEA & Gelfoam \\
$58 /$ female & Post MVR and TVR & Warfarin & Prolonged PT/INR & Right IEA & Gelfoam \\
\hline
\end{tabular}

Gelfoam SIEAH, spontaneous inferior epigastric artery hemorrhage; MVR, mitral valve replacement; TVR, tricuspid valve replacement.
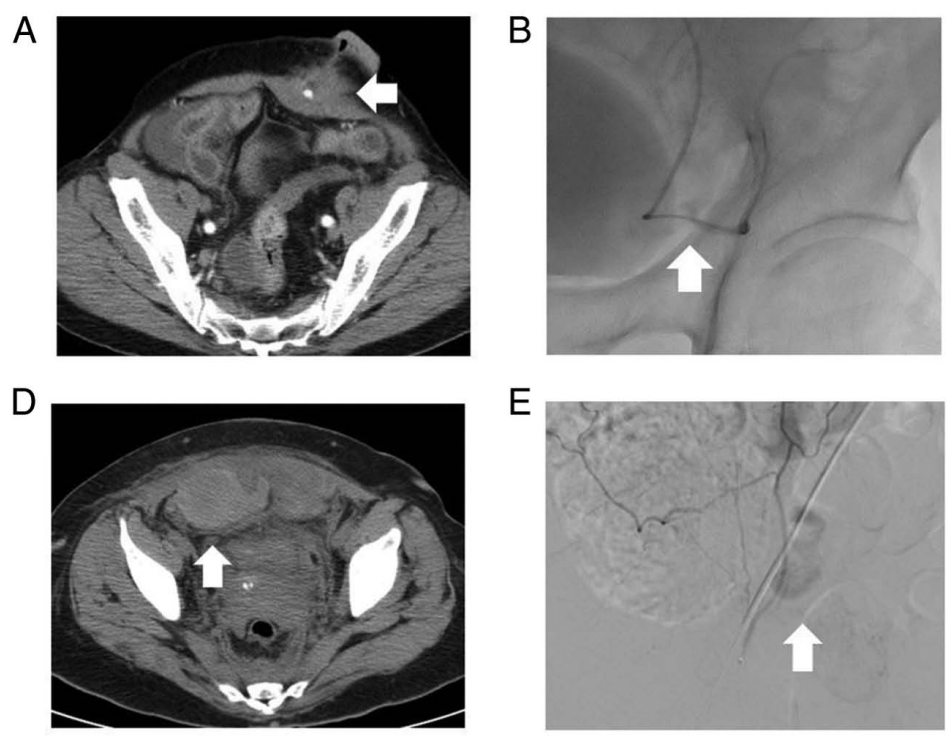

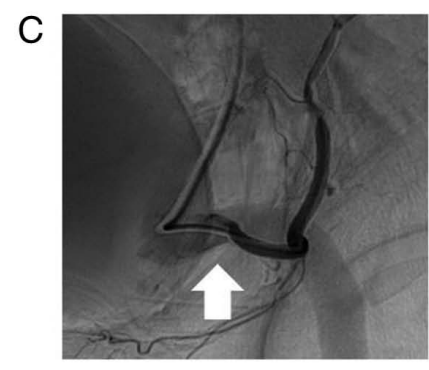

$\mathrm{F}$

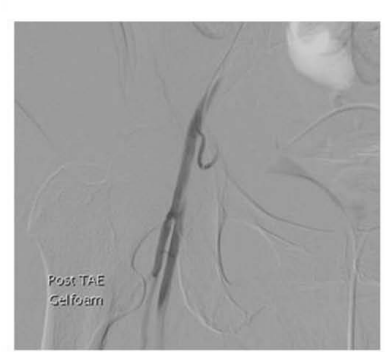

Figure 1. (A) Tissue swelling and hemorrhage from the incision wound with contrast extravasation observed (arrow). (B) Angiography confirmed active bleeding from the left inferior epigastric arterial branches (arrow). (C) No contrast extravasation following superselective embolization (arrow). (D) Enlargement of the enhanced densities over the bilateral lower abdominal wall and more prominent right-side rectus abdominis (arrow). (E) Angiography revealed active bleeding point from the right inferior epigastric artery (arrow). (F) Successful superselective embolization without contrast extravasation.

Computed tomography (CT) revealed contrast extravasation on the left lower abdominal wall (Fig. 1A). Subsequent angiography revealed active bleeding from the left IEA branches (Fig. 1B). To achieve hemostasis, superselective catheterization and conducted transarterial embolization we performed using Gelfoam (SURGIFOAM ${ }^{\circledR}$, Ethicon). Bleeding was successfully terminated following the procedure (Fig. 1C). Owing to the state of shock of the patient, he was referred to the surgical intensive care unit. The patient was discharged on post-operative day 23 . Written informed consent was provided by the patient.

Case 2. A 58-year-old female with a history of mitral and tricuspid valve replacement received anticoagulant therapy with warfarin ( $3 \mathrm{mg} /$ day). She visited the Outpatient Department at Kaohsiung Medical University Chung-Ho Memorial Hospital and complained of a productive cough and abdominal pain. A physical examination revealed a tender mass on the lower abdominal area. Laboratory data revealed mildly prolonged PT/INR as 1.44 (range, 0.85-1.15). Initially, conservative treatment was applied with bed rest and ice pack application. However, the symptoms persisted, and the hemoglobin level decreased from 10.7 to $9.3 \mathrm{~g} / \mathrm{dl}$. CT findings revealed hematoma in the bilateral lower abdominal wall with persistent bleeding (Fig. 1D). Subsequent angiography revealed contrast extravasation from the right IEA (Fig. 1E). Superselective embolization was performed with Gelfoam (SURGIFOAM ${ }^{\circledR}$, Ethicon) pledgets (Fig. 1F). Post-operatively, the recovery course was uneventful. The patient was discharged on postoperative day 7. Written informed consent was provided by the patient. A summary of the 2 cases is presented in Table I.

\section{Discussion}

RSH accounts for $1-2 \%$ of acute abdominal syndrome cases $(2,5,8,11)$. Although its incidence is rare, certain studies have reported a high mortality rate of up to $25 \%$ with anticoagulant therapy $(2,4,11,12)$. The etiology of RSH includes trauma, intensive muscle contractions, and iatrogenic and anticoagulant therapy $(2,3,5,12,13)$. Anticoagulant therapy is one of the major risk factors of RSH (14). In a previous study, Cherry and Mueller reviewed $126 \mathrm{RSH}$ cases and found that $25 \%$ of the patients received anticoagulant therapy (14). SIEAH is a relatively rare condition of RSH $(2,5)$. The pathophysiology of SIEAH remains unclear. One hypothesis is unrecognized minor trauma, such as cough, sneezing, or passive joint motion $(8,9,11)$. Patients with an advanced age are more susceptible to suffering from SIEAH. The cause may be rectus sheath weakness or small vessel atherosclerosis, 
or both $(8,9,12,13)$. Nevertheless, anticoagulant therapy is strongly associated with SIEAH $(5,8,11,13)$.

IEA pseudoaneurysm rupture is also a rare etiology of RSH (15-17). The majority of cases of IEA pseudoaneurysm rupture are iatrogenic, such as the extraction of the retention suture, surgical trauma, drain tube insertion or removal, and therapeutic paracentesis $(15,16)$. Color Doppler sonography can present 'to-and-fro' flow (16). CT and angiography can also reveal pseudoaneurysm and bleeding (15-17). In the present study, in case 1, the patient exhibited RSH on post-operative day 10. Moreover, the left para-midline incision wound was just beyond the extravasation point (Fig. 1A). Therefore, iatrogenic IEA pseudoaneurysm rupture can be a possible cause of RSH. However, angiography revealed no signs of pseudoaneurysm, only contrast extravasation. Thus, it was considered that the bleeding episode was SIEAH related to anticoagulant therapy.

The most common presentation of SIEAH is abdominal pain $(2,8,11,12)$, which can become aggravated with the patient's movements $(2,3,5,8)$. The typical finding during physical examination is an abdominal mass, which does not cross the middle abdomen $(2,3,5,7,8)$. Peritoneal signs, such as muscle guarding or rebounding pain, may be positive; therefore, the presentation can mimic that of acute abdominal syndrome $(3,7,8,11,12)$. Laboratory data may reveal normal coagulation function even with active bleeding $(2,11)$. Bedside sonography can provide some information on differential diagnosis; however, CT is the most effective imaging modality $(5,12)$. Both sensitivity and specificity can reach $100 \%(2,3,5,11,12)$.

Since hematoma caused by SIEAH is confined to the rectus sheath, bleeding can be self-limited. Thus, conservative treatment with compression, the use of ice packs and blood transfusion should be the appropriate initial treatments $(2,3,5,12,13)$. Invasive management, such as surgical intervention with hematoma removal and ligation of the bleeding vessel, has been the most standard treatment, particularly for correcting hemodynamic instability $(2,3,5,7,12)$. However, locating the bleeding point in hematoma and muscle fiber is difficult $(9,10)$. Moreover, releasing the pressure of hematoma may aggravate bleeding (9). In patients with severe comorbidities, old age, or other associated injuries, the surgical intervention may result in several complications. Hence, in some selected cases, a minimally invasive procedure, such as TAE, may be the choice of treatment for SIEAH $(3,7,8,11)$. Some studies have suggested that the shock status, such as hypotension and tachycardia, is not a contraindication of TAE $(3,10)$. If the diagnosis of SIEAH is definite, embolization can be performed to achieve hemostasis $(3,8,10,11)$.

In patients with SIEAH with unstable hemodynamic status, to date, data suggesting that TAE is more suitable than traditional open surgery are lacking $(10,12)$. However, it is suggested that with the improvement of the imaging system and equipment, for selected patients with anticoagulant therapy, TAE may be the optimal treatment, as with Case 1 in the present study, for example. In a patient with old age and acute myocardial infarction, TAE may be superior to open surgery as the initial treatment.

In conclusion, the standard use of anticoagulant or antiplatelet agents may induce severe bleeding episodes, such as SIEAH. Although the condition is rare, it can be potentially life-threatening. The diagnosis is mainly dependent on CT findings. Surgery is a feasible treatment; however, it has some limitations. In some selected patients receiving anticoagulant therapy, TAE, as a minimally invasive procedure, may be the preferred treatment for SIEAH.

\section{Acknowledgements}

Not applicable.

\section{Funding}

No funding was received.

\section{Availability of data and materials}

Data sharing is not applicable to this article, as no datasets were generated or analyzed during the current study.

\section{Authors' contributions}

YCC was involved in the management of the cases and the preparation of the manuscript. CLH was involved in the management of the cases. MCS perform the transcatheter arterial embolization. JYW was involved in the management of the cases and the critical appraisal and review of the manuscript. All authors have read and approved the final manuscript.

\section{Ethics approval and consent to participate}

Written informed consent was provided by the patients.

\section{Patient consent for publication}

The patients provided written informed consent for the patient information to be published.

\section{Competing interests}

The authors declare that they have no competing interests.

\section{References}

1. Romic I, Pavlek G, Mance M, Romic M and Moric T: Fatal case of spontaneous rectus sheath hematoma caused by anticoagulant and steroid therapy during hospital admission. Visc Med 34: 225-227, 2018.

2. Ben Selma A and Genese T: Spontaneous rectus sheath hematoma: An uncommon cause of acute abdominal pain. Am J Case Rep 20: 163-166, 2019.

3. Azharuddin M, Gupta M and Maniar M: Subcutaneous heparin leads to rectus sheath hematoma: A rare complication. Cureus 10: e2769, 2018.

4. Stillman K and Kellar J: Rectus sheath hematoma: An unfortunate consequence of novel anticoagulants. West J Emerg Med 16: 420-421, 2015

5. Takahashi K, Nihei T, Aoki Y, Nakagawa M, Konno N, Munakata A, Okawara $\mathrm{K}$ and Kashimura $\mathrm{H}$ : Spontaneous rectus sheath hematoma associated with warfarin administration: A case report. J Rural Med 14: 245-248, 2019.

6. Ueno T, Nakamura T, Hikichi H, Arai A, Suzuki C and Tomiyama M: Rectus sheath hematoma following intravenous thrombolysis with recombinant tissue plasminogen activator for cerebral infarction: A case report. J Stroke Cerebrovasc Dis 27: e237-e238, 2018.

7. Wick MC, Klocker J, Grundtman C, Jaschke W and Chemelli AP: Transcatheter embolization for the management of acute active inferior epigastric artery hemorrhages. J Endovasc Ther 20: 561-567, 2013. 
8. Buffone A, Basile G, Costanzo M, Veroux M, Terranova L, Basile A, Okatyeva V and Cannizzaro MT: Management of patients with rectus sheath hematoma: Personal experience. J Formos Med Assoc 114: 647-651, 2015.

9. Sharafuddin MJ, Andresen KJ, Sun S, Lang E, Stecker MS and Wibbenmeyer LA: Spontaneous extraperitoneal hemorrhage with hemodynamic collapse in patients undergoing anticoagulation: Management with selective arterial embolization. J Vasc Interv Radiol 12: 1231-1234, 2001

10. Basile A, Medina JG, Mundo E, Medina VG and Leal R: Transcatheter arterial embolization of concurrent spontaneous hematomas of the rectus sheath and psoas muscle in patients undergoing anticoagulation. Cardiovasc Intervent Radiol 27: 659-662, 2004

11. Hatjipetrou A, Anyfantakis D and Kastanakis M: Rectus sheath hematoma: A review of the literature. Int J Surg 13: 267-271, 2015.

12. Salemis NS: Spontaneous rectus sheath hematoma presenting as acute surgical abdomen: An important differential in elderly coagulopathic patients. Geriatr Gerontol Int 9: 200-202, 2009.

13. Aktas H, Inci S, Dogan P and Izgu I: Spontaneous rectus sheath hematoma in a patient treated with apixaban. Intractable Rare Dis Res 5: 47-49, 2016.

14. Cherry WB and Mueller PS: Rectus sheath hematoma: Review of 126 cases at a single institution. Medicine (Baltimore) 85: 105-110, 2006.
15. Sobkin PR, Bloom AI, Wilson MW, LaBerge JM, Hastings GS, Gordon RL, Brody LA, Sawhney R and Kerlan RK Jr: Massive abdominal wall hemorrhage from injury to the inferior epigastric artery: A retrospective review. J Vasc Interv Radiol 19: 327-332, 2008.

16. Nichols-Totten K, Pollema T and Moncure M: Pseudoaneurysm of the inferior epigastric artery: A rare complication of laparoscopic ventral hernia repair. Surg Laparosc Endosc Percutan Tech 22: e25-e27, 2012.

17. Ruiz-Tovar J, Rubio M, Conde S, Morales V and MartinezMolina E: Inferior epigastric artery pseudoaneurysm: Complication of surgical drain insertion. ANZ J Surg 78: 1139, 2008.

(i) $(-)$ This work is licensed under a Creative Commons Attribution-NonCommercial-NoDerivatives 4.0 International (CC BY-NC-ND 4.0) License. 